\title{
Early Exposure to County Income Mobility and Adult Individual Health in the United States
}

\author{
Sebastian Daza \\ Center for Demography and Ecology \\ University of Wisconsin-Madison \\ sdaza@ssc.wisc.edu \\ Alberto Palloni \\ Center for Demography and Health of Aging \\ University of Wisconsin-Madison
}

August 11, 2020

\footnotetext{
* The research on which this paper is based was supported by the National Institute on Aging via research project grants (R01 AG016209 [PREHCO], R03 AG015673, R01 AG018016, and MERIT award R37 AG025216), and by a Fogarty International Center award for Global Research Training in Population Health (D43 TW001586). The University of Wisconsin-Madison researchers are supported by core grants to the Center for Demography and Ecology, University of Wisconsin (R24 HD047873) and to the Center for Demography of Health and Aging, University of Wisconsin (P30 AG017266), and a small grant for research using PSID Data through the National Institute on Aging (P01AG029409).
} 


\section{Abstract}

Despite substantial research, drivers of the widening gap in life expectancy between rich and poor in the U.S. - the so-called longevity gap - remain unknown. Recent research has suggested that contextual income mobility (e.g., county-level socioeconomic mobility) may play an essential role in explaining the longevity gap. Previous studies - based mostly on aggregate and cross-sectional individual data - show an association between county income mobility and county mortality and individual's health. However, inferring individual effects from aggregate (county-level) data can be problematic (i.e., ecological fallacy), and measuring exposure to income mobility using the county where respondents currently live or die, might overlook the selection process associated with residential mobility. This paper aims to extend previous research by estimating the effect of average exposure to mobility regimes during childhood and adolescence on adult health using longitudinal data and accounting for selection into counties over time (i.e., residential mobility). We use both the National Longitudinal Survey of Youth 1997 (NLSY97) and the Panel Study of Income Dynamics (PSID) with geocoded data to assess the link between county-level income mobility (Chetty's estimates), behaviors (smoking) and health conditions and status (self-reported health, BMI, depressive symptoms). Furthermore, we use cohorts optimally match Chetty's estimates of income mobility in the U.S. (1980-1982) and account for selection and time-varying confounders using marginal structural models (MSM). Overall, we provide a more precise test of the hypothesis that childhood exposure to income mobility regimes may determine health status through behavior (i.e., smoking) later in life and contribute to longevity gaps. 


\section{Introduction}

The growing life expectancy gap by income represents a fundamental challenge for health policy in the U.S. The best performing U.S. counties have life expectancies that are 20 years greater than the poorest performers. Recent work by Chetty and colleagues (Chetty et al., 2016) shows that the difference in life expectancy (at age 40 ) between the richest $1 \%$ and poorest $1 \%$ in the United States is 14.6 years for men and 10.1 years for women. Also, between 2001 and 2014, life expectancy has increased 2.34 years for men in the top $5 \%$ of the income distribution and 2.91 years for women, but only 0.32 years for men in the bottom $5 \%$ of the distribution and 0.04 years for women. These gaps are substantial - representing about $35 \%$ of remaining life expectancy at age 40 among men and $25 \%$ for women.

A large body of recent research demonstrates that neither access to medical care nor socioeconomic factors fully explain observed geographic or income disparities in longevity. The search for drivers of the longevity gap has led scholars to suggest that contextual income mobility — defined as the ability of individuals to exceed their parents' income - may play an essential role in explaining health disparities (Daza \& Palloni, 2018; Venkataramani et al., 2016, Venkataramani et al., 2015; Venkataramani et al., 2020). For instance, low-income mobility may harm health by raising despair and diminishing the motivation to engage in healthy behaviors. These effects would be distinct to the consequences of income inequality for health. Individuals living in areas characterized by similar high degrees of income inequality may experience different probabilities of income mobility - and therefore may have different impacts on health outcomes. While the association between income inequality and health has been studied over the last 20 years, recent work states that its contribution to disparities in longevity may be small (Chetty et al., 2016). In contrast, the health consequences of economic mobility remain understudied. This gap in the literature is particularly salient given emerging evidence of falling income mobility in the U.S., especially among the same birth cohorts currently experiencing divergence in their life expectancy (Chetty et al., 2017).

Previous evidence on the link between income mobility and health comes mostly from the analysis of aggregate data (Daza \& Palloni, 2018; Venkataramani et al., 2015; Venkataramani et al., 
2020), and individual cross-sectional surveys (Venkataramani et al., 2016). We identify at least three main limitations of previous research. First, associations observed at the aggregate (e.g., county-level data) might not be kept at the individual level when most of the mechanisms proposed in the literature consist of individual processes (i.e., ecology fallacy), not aggregate ones (Daza \& Palloni, 2018). Consequently, a direct way to assess the hypothesis on the link between contextual income mobility and health would require individual-level data. Second, as the neighborhood effects literature has pointed out (Sampson et al., 2002; Wodtke et al., 2016; Wodtke et al., 2011), residential mobility might produce spurious associations between contextual variables and individual outcomes. To obtain unbiased estimates of the effect of contextual income mobility on health outcomes, we need either experimental or longitudinal data that allow us to adjust for selection associated with residential mobility. Lastly, previous research has not defined clearly when exposure to a place's income mobility during the life course would have significant consequences for health. Are the consequences the same whether the exposure was in childhood or at age 40? The theory suggests that this would not be the case. Thus, it is necessary to define clearly what exposure is and how it is measured to correctly interpret the associations found in the data and avoid over-interpreting spurious relationships.

Our study extends previous research by estimating the effect of average exposure during childhood and adolescence on health outcomes and behaviors measured during young adulthood (the early thirties and forties) using longitudinal data. We employ both the NLSY97 and PSID with geocode data to assess the link between county-level income mobility (Chetty et al. 2014's estimates) and health outcomes and behaviors such as self-report health, BMI, depression, and smoking. Also, we use data that match better the cohorts used by Chetty to estimate of income mobility in the U.S. at the county level (i.e., children born between 1980 and 1982), account for selection associated with residential mobility over time, and adjust for time-varying confounders using marginal structural models (MSM). Thus, we provide a more precise assessment of the hypothesis that exposure to income mobility may determine health later in life and explain the longevity gap. 


\section{Individual mechanisms}

We briefly discuss potential individual mechanisms that would generate an association between the place's income mobility and health. First, we notice we are examining the relationship between a place's income mobility (i.e., an aggregate property of the stratification system) and health outcome (i.e., an individual trait), not the relationship between individuals' lifetime income mobility and adult health - a problem studied in a large and distinguished body of research (Blane et al., 1999; Blane et al., 1993; Chandola et al., 2003; Fox et al., 1982; Illsley, 1955; Solon, 1992) 11 It is, of course, possible that individuals' experiences of occupation or SES mobility are also influenced by the prevailing aggregate regime of income mobility. Indeed, these experiences may be one of many other pathways through which a place's income mobility and individual health are linked. However, in this paper, we focus on the total effects of place's income mobility on individual health, not in the precise empirical identification of mediating pathways.

We argue that a link between places' income mobility and mortality could exist if communities with higher income mobility host social and economic environments that reduce mortality risks relative to communities with lower income mobility, independently of the income level and income inequality. Individuals who occupy the most vulnerable and exposed social positions within unequal communities may be comparatively better off when facing advantageous income mobility prospects than when they do not. Just as individuals who command lower incomes in communities with more equitable income distributions may experience better health than individuals with similar incomes in societies with higher income inequality, persons that occupy lower-ranked positions in societies with higher income mobility may enjoy better health than counterparts in societies with more rigid stratification systems.

Theoretically and empirically, communities can be classified in different combinations of income mobility and inequality, where each combination is characterized by a health and mortality

\footnotetext{
${ }^{1}$ The bulk of this literature is concerned with the long-run impact of early occupational (career) shifts or the shortrun effects of late occupational (career) shifts. This work is based on empirical research that focuses on patterns of relationships between individuals' occupation (or SES status broadly conceived) at an early point in adult life and subsequent older adult health and mortality.
} 
profile.$^{2}$ The standard conjecture is that indicators of mortality and health will be more beneficial in communities with less inequality than those with high inequality. We extend that conjecture by stating that, given a level of income inequality, better health and mortality conditions will be experienced by community members with higher income mobility. Following a neo-material reinterpretation of the effect of income inequality on health (Lynch et al., 2004), we can argue that the place's income mobility by itself is not the root of health disparities, but only a manifestation of a cluster of conditions that affect population health, so that the association between income inequality and health would be contingent on people's resources at the individual and community levels. Accordingly, to estimate the independent effect of inequality or mobility on health, we would need to account for material factors at the community level that might confound the association between health and stratification characteristics, taking the precaution of not adjusting for mediators of the causal process between income mobility and health.

We identify at least four pathways that might produce a link between income mobility and health. First, the association between aggregate income mobility and individual health may be the outcome of a composition effect. Namely, places with higher income mobility contain a population composition biased toward individuals who experience socioeconomic mobility. In this case, the association between a stratification trait and individual experiences of health and mortality would reflect the influence of individual residential mobility patterns and selection.

Second, exposure to income mobility during childhood and adolescence may be influential as vehicles that establish relations between income inequality, income mobility, and adult health. There is evidence that individuals' early conditions and upbringing matter greatly for adult health and mortality disparities (Case et al., 2002; Palloni et al., 2009). Thus, some of the health differentials between men in low and high ranking positions initially attributable to chronic stress among those in subordinate positions (Marmot, 2004; Sapolsky, 2005) may be rooted in antecedent health conditions sculpted early in life (Case \& Paxson, 2011). For instance, individuals can experi-

\footnotetext{
${ }^{2}$ Our data, for instance, show communities (counties) with both an unequal income distribution and flexible mobility regimes (high income mobility), but also communities with a generous income distribution and high social rigidity.
} 
ence sensitive and critical windows for the acquisition of cognitive and non-cognitive abilities during early stages of socialization, which, in turn, are the foundation of skills acquired later in life (Cunha \& Heckman, 2009; Heckman, 2007; Knudsen et al., 2006; Shonkoff et al., 2009). Some of these traits involve the development of outlooks and attitudes that influence investments in skill acquisition and health, including propensities to adhere to health-related behaviors. Thus, behaviors critically associated with modern chronic illnesses, such as smoking uptake and desistance, alcohol consumption, substance abuse, choices of diet and physical activity, would be determined, in part, by capabilities sculpted early in life. Early avoidance of unhealthy behaviors has large pay-offs in adulthood because these behaviors are closely related and reinforce each other, the physiological and psychological damage they produce are accumulated over time, and they all are strongly non-reversible. Early adoption of healthy behaviors is facilitated by socialization that emphasizes robust future outlooks, self-confidence, and self-reliance, beliefs in the neutrality and fairness of social reward allocation systems, hopefulness and optimism, and incentives to succeed. These are all traits that reduce time discounting so that the addition of one year of healthy life in the future of an individual is endowed with significant rewards and returns (Grossman, 1972, 2000).

We also know from empirical research that negative affect, chronic stress, subordination, bleak future outlooks associated with poverty lead to increases in time discounting (Haushofer \& Fehr, 2014). Higher time preferences favor resistance to the adoption of behaviors that may yield immediate rewards but are health-damaging and discourage those that have a more distant and elusive pay-off but are health-preserving (Eigsti et al., 2006; Schlam et al., 2013). This mechanism alludes to influences that shape environments during critical stages of individuals' upbringing. The conjecture is that a places' income mobility regime is powerful enough to shape those environments. Nevertheless, so are individual's ancestral income mobility experiences, particularly parental and, possibly, grand-parental socioeconomic mobility. Strictly speaking, these are mechanisms that can be properly identified only if we simultaneously observe the influences of a place's aggregate income mobility and individuals' familial income mobility experiences.

Lastly, communities with low-income mobility may distort opportunities and incentives, re- 
inforce unequal allocation of favorable traits, undervalue public institutions that contribute to the formation of skills with a high wage premium and, many of them support non-meritocratic reward allocation strategies. These community properties directly influence the suite of opportunities available to individuals and shape how parents socialize children and favor (discourage) the adoption of positive outlooks and the value of skill acquisition. Rigid or weak income mobility fosters individual hopelessness, despair, mistrust, disbelief in a level playing field for all, weaken aspirations, and, more generally, diminishes the value of adopting attitudes and behaviors that promote good health. This is in line with the hypothesis proposed by Case and Deaton (2020), who reported the fastest-rising death rates of causes such as suicides, drug overdoses, and alcoholic liver disease in the U.S., especially among those without a bachelor's degree. These self-inflicted deaths have been designated as deaths of despair as they affect those who face economic, social, or psychological adversities, hopelessness, and lack of well-being. We suggest that these population's adversities, in addition to a rigid or weak income mobility regime, would decrease the adoption of healthy behaviors, affecting not only the current generation but also those to come.

Based on these mechanisms, especially the hypothesis that early exposure to a given income mobility regime has substantial consequences for adult health, we examine whether exposure to a given mobility regime during childhood and adolescence impacts health indicators later in life, after adjusting for residential mobility selection and time-variant confounders. Thus, we extend

previous research by carrying out a more precise assessment of the link between contextual income mobility and health.

\section{Data}

Our data result from combining different sources. The first is the Health Inequality Project Data (HIPD) created by Chetty and colleagues (Chetty et al., 2016). Those data - the result of linking 1.4 billion tax records to Social Security Administration records - contain information on income 
for the period 1999-2014 by U.S. counties and commuting zones $3^{3}$ The HIPD also include statistics of the income distributions and two indicators of income mobility derived from measures of the association between incomes of children born between 1980 and 1982 and their parents' income..$^{4}$ First, we use the index of relative mobility (IRM or rank-rank slope) at the county level that is the correlation between children's income rank - within a birth cohort - and their parents' income rank.$^{5}$ The relative income mobility indicator ranges between -1 and 1 , and larger values correspond to lower income mobility (i.e., higher rank-rank correlation between parents' and child's income). We also use an absolute upward mobility score or "the mean rank (in the national income distribution) of children whose parents are at the $25^{\text {th }}$ percentile of the national parent income distribution" (Chetty et al., 2014, p. 7) ${ }^{6}$ Absolute upward income mobility ranges from 0 to 1 , and higher values correspond to larger income mobility. To facilitate interpretation, we multiply the upward mobility score by -1 so that the meaning and expected association of relative and absolute income mobility with health are the same. Finally, we use the Gini coefficient as an indicator of income inequality.

The second database is the National Longitudinal Survey of Youth 1997 (NLSY97), a nationally representative sample of 8,984 American youth born between 1980 and 1984. Surveys were conducted annually, beginning in 1997 when the youth were between 12 and 18 years of age. In the first round, both the eligible youth and one of their parents were administrated personal interviews. The restricted NLSY97 geocoded data file contains information on the geographic residence of each respondent since age 12, allowing us to merge it with Chetty's county level income mobility measures. Importantly, the NLSY 97 sample matches the cohorts of the core sample used by

\footnotetext{
${ }^{3}$ Chetty et al. (2014)'s core sample data include children who (1) have a valid Social Security number or individual taxpayer identification number, (2) were born between 1980 and 1982, and (3) are U.S. citizens as of 2013. There are approximately 10 million children in the core sample.

${ }^{4}$ We use a permanent-resident version of income mobility measures, that is, parents who stay in the same counties between 1996-2012. Note that children who grow up in a county may have out-migrated as adults.

${ }^{5}$ Rank-rank slopes (or Spearman's correlation) have proved to be quite robust across specifications and highly suitable for comparisons across areas (Chetty et al., 2014). Canonical measures of relative mobility, such as intergenerational income elasticity (of child income relative to parents' income) tend to be sensitive to changes in inequality across generations.

${ }^{6}$ Although at the national level both the relative and absolute measure of mobility provide similar information, when studying small areas a child's rank in the national income distribution would be an absolute outcome because income in a given area has little impact on the national distribution.
} 
Chetty et al. (2014) (1980-1982), so we can align better the timing of early exposure to the place's income mobility. This under the assumption that the income mobility of this cohort measures the socioeconomic mobility regime to which this generation was exposed early in life and that may affect their health later. After merging the two databases, we kept 8,810 NLSY97 respondents. Only 174 respondents $(2 \%)$ were removed from the analytic sample because income mobility information did not match the NLSY97 data. The total number of counties matched was 1607. Figure S1 in the Methodological Supplement shows counties included in the NLSY97 sample by income mobility, inequality, and the log of county's population. Figure $\mathrm{S} 1$ shows that counties included in the sample have a larger population, less variability and extreme values in the income mobility measures than the counties excluded. The distribution of income inequality is much more symmetric by population size. Although our analyses adjust for sampling weights and county's population size, we note our sample differs from the county composition of previous aggregate and individual level studies that have a higher county coverage. The potential consequences of this coverage difference for our results are discussed later in the Conclusion and discussion section.

The third database is the Panel Study of Income Dynamics (PSID), a nationally representative sample of U.S. men, women, children, and their families followed for more than 40 years. The PSID began interviewing a sample of about 5,000 families in 1968 and were re-interviewed each year through 1997 when the data collection became biennial. Similarly to the NLSY, restricted geographic data allow us to merge individual records with county income mobility measures. Unlike the NLSY 97, the PSID data permit us to estimate the effect of exposure to contextual mobility from birth to age 20. However, we lose statistical power because the number of respondents who match the Chetty et al. (2014)'s cohort is smaller. For instance, between 1975 and 1985, the PSID panel had 4,771 newborns $!^{7}$ Of these, 2,358 were the reference person or spouse/partner of the household at any time during their participation in the panel ${ }^{8}$ Although that cohort does not match

\footnotetext{
${ }^{7}$ These newborns are PSID gene respondents. All 1968 sample members have the PSID gene, and they are followed in all subsequent waves across their entire lives, regardless of where they live. All individuals born to or adopted by somebody with the PSID gene acquires the gene themselves, and therefore are followed. Respondents who also were the household head or spouse/partner were asked most of our health outcomes overtime and had less missing data.

${ }^{8}$ The outcome variables included in our analysis were mostly asked to reference persons and their spouses or
} 
exactly the cohort used by Chetty et al. (2014), it offers a reasonable approximation to the mobility regime exposure of that generation, provided income mobility does not change dramatically before 1980-82. After merging PSID and HIPD databases, we obtained 2,273 respondents.9. Only 85 respondents $(4 \%)$ were removed because income mobility information did not match the PSID data. The total of counties matched was 1120, and the distribution of counties by income mobility, inequality, and population size looks similar to the NLSY97. ${ }^{10}$ Even though the PSID analytical sample is considerably smaller, we think it is worth to estimate the effects of exposure from birth to age 20 years, and compare those results with the NLSY97.

Using different longitudinal data sources provides a broader picture to examine our research questions. It also offers a more precise definition of exposure to contextual income mobility as both of studies track respondents' county of residence during early life and over a relatively long period of time.

\section{Analytical Strategy}

This paper aims to estimate the effect of average exposure to county income mobility during childhood and adolescence on health outcomes such as smoking, BMI, self-reported health, and mental health during young adulthood. The key independent variable is the average income mobility exposure between ages 12 and 20 in the case of the NLSY97, and ages 1 to 20 for the PSID. Outcomes, in contrast, were measured during the last NLSY97 and PSID waves when respondents were in their thirties or forties. As a benchmark, we used both relative and absolute income mobility and estimated the effect of average county income inequality exposure (i.e., Gini coefficient), to compare the magnitude and direction of the associations. We used residualized income mobility and inequality scores from a county-level regression model that adjusted for characteristics

partners. That is why we only consider respondents who were a reference person or partner at least once during the observation period.

${ }^{9}$ We also used the PSID Well being and Daily Life Supplement 2016 complete missing data of variables such as depression symptoms in the PSID core database.

${ }^{10}$ Due to disclosure rules for restricted PSID data, we cannot show the scatter plot of individuals by county. 
such as population size, proportion of African-Americans, average household income, and income inequality (or income mobility). For completeness, we show the results with non-residualized exposure treatments in the Methodological Supplement.

We modeled the health outcomes as a function of duration-weighted exposures to different levels of county mobility regimes. By using inverse probability of treatment weighting (IPT), we emulated a counterfactual scenario in which we compared children with the same combination of observed covariate values during the exposure time, who did not select systematically into different county mobility regimes. Thus, we adjusted for confounding by time-varying covariates that might be affected by past treatment (Hernán et al., 2002; Hernán \& Robins, 2006; Hernán et al., 2000; Wodtke et al., 2011), and generated a pseudo-population in which treatment was no longer confounded with measured covariates. Weights balance treatment assignment across prior confounders and give more or less weight to children with covariates histories that are underrepresented (or over-represented) in their current treatment group. To reduce the variability of weights, we used stabilized IPT weights (Hernán et al., 2000; van der Wal \& Geskus, 2011). As the estimation of stabilized weights includes time-invariant covariates in the numerator and denominator, final outcomes models need to condition on time-invariant covariates in order to obtain unbiased estimates of the treatment.

As a sensitivity analysis, and because IPT weights using a continuous treatment are more sensitive to misspecification and outliers (Naimi et al., 2014; Thoemmes \& Ong, 2016), we estimated weights for both continuous and categorical scores of income mobility and inequality. While we used linear regression in the first case, we ran ordinal logistic regressions to estimate the probability of exposure to county income mobility quintiles. Finally, following the strategy suggested by Dugoff et al. (2014), we included the sampling weights when computing IPT weights, multiplied them, and considered survey design variables (i.e., strata, clusters) and compound weights when estimating exposure models. 


\subsection{NLSY97}

The NLSY97 has information of respondents' location (county) since age 12, so the exposure to county income mobility between ages 12 and 20 can be defined as:

$$
\frac{\sum_{i=12}^{20} \text { county income mobility }}{8}
$$

We employed several covariates to adjust for potential confounding of county income mobility effects on health outcomes. Time-invariant covariates include race, gender, parents' education (years), age by the end of the study (categorical variable), the number of residential moves by age 12, the Armed Services Vocational Aptitude Battery (CAT-ASVAB) score, and mother's age at birth. Time-variants covariates, in turn, are inflation-adjusted family income (log), family size, the cumulative number of county changes, whether parents are employed and married, self-report health status, the number of days smoked in the last month, and BMI ${ }^{11}$ Table $\mathrm{S} 5$ in the Methodological Supplement shows descriptive statistics of the variables included in our models.

To estimate stabilized IPT weights in Time 1 (Age 12), we employed only time-invariant covariates. From Time 2 to 8 , we used both time-invariant, baseline, and lagged time-variant covariates so that weights for later time points included all previous variables. ${ }^{12}$ Table $S 7$ in the Methodological Supplement shows descriptive statistics for the stabilized IPT weights for both continuous and categorical exposure treatments.

We used multiple imputation with multilevel models to address both item-specific non-response and attrition. ${ }^{13}$ By design, respondents interviewed for the first time after their $12^{\text {th }}$ birthday do not have information between age 12 and the age of the first interview. About $32 \%$ of the NLSY97

\footnotetext{
${ }^{11}$ The number of cigarettes smoked during the last month was asked only until 2011 , that is why, we decided to use the number of days smoked during the last month. Moreover, rounds 4, 6, 8, 10, 12, 14 and 17 of the NLSY97 include a five-item short version of the Mental Health Inventory (MHI-5) to screen for depressive symptoms. Respondents reported the frequency of being nervous, feeling calm and peaceful, feeling downhearted and blue, being happy, and feeling so down in the dumps that nothing could cheer them up using a four-point scale to rate the frequency of their feelings. Because the MHI-5 was only measured in later rounds of the survey, we do not include that scale as a time-variant covariate.

${ }^{12}$ Details on model specification are available in https://github.com/sdaza/dissertation/tree/master/ch03.

${ }^{13}$ See van Buuren (2018) for an example of selective drop-out correction through multiple imputation.
} 
respondents had full exposure information (i.e., eight interviews from age 12 to 20). On average, respondents reported 6.5 years (out of 8 ), and only $7 \%$ of the sample participated in four or fewer years (e.g., older interviewees). When the county of residence was missing over the follow-up period, we imputed lost counties using most recent or earliest county of residence based on the evidence that most people do not change their county of residence often 14 Matched NLSY97 counties with the HIPD data cover, on average, 5.9 out of 8 years of exposure, and only $4 \%$ of the sample have less than three years. At the end, we imputed missing records by creating 20 multiple imputed data-sets. 15

We implemented different outcome models depending on the nature of the dependent variable. We estimated ordinal logistic regression for the effect of income mobility and inequality on selfreported health status (poor, fair, good, very good, excellent), Generalized linear models (GLM) for BMI and depression symptoms, logistic regression in the case current smoking status, and quasiPoisson models (also called over-dispersion with quasi-likelihood) for the number of days smoking in the last month. Outcome models adjusted only for baseline and time-invariant covariates and took into account sampling design variables (strata, clusters) and weights.

\subsection{PSID}

The PSID sample includes newborns. Thus, we could define the average exposure to county income mobility from age 1 to 20 as:

$$
\frac{\sum_{i=1}^{20} \text { county income mobility }}{20}
$$

We included a relatively similar set of covariates as the NLSY97 sample. Time-invariant covariates involved race, gender, age by the end of the study, mother's age and marital status at birth,

\footnotetext{
${ }^{14}$ According to the U.S. Census Bureau migration estimates (Current Population Survey and Annual Social and Economic Supplement 1948-2019), 16\% of the U.S. population changed their residence between 1999 and 2000. Of those, 56\% remain in the same county (see https://www.census.gov/data/tables/time-series/demo/geographic-mobility/ historic.html). In practice, we implemented the Last Observation Carried Forward (LOCF) and Next Observation Carried Backward (NOCB) methods.

${ }^{15}$ For a discussion and assessment of our multiple imputation models, see the Methodological Supplement
} 
and weighed less than 55 pounds at birth. In turn, time-variants covariates were inflation-adjusted family income (log), family size, the cumulative number of county changes, head of household education, whether the head was employed, married, and owns the house where that family was living. Outcomes included self-report health status, BMI, depression, current smoking, and number of cigarettes smoked during the last month. 16 Unlike the NLSY97, PSID outcome variables were not measured systematically during the exposure period. Thus, we decided not to use outcomes as time-variant predictors when estimating IPT weights. Table S6 in the Methodological Supplement shows descriptive statistics of the variables used.

We followed the same procedure described in the previous section to estimate IPW weights. First, we computed stabilized IPT weights for Time 1 by including only time-invariant covariates. Then, we created weights from Time 2 to 20 using time-invariant and lagged time-variant covariates so that weights for subsequent time points include all previous variables. Table S8 in the Methodological Supplement shows descriptive statistics of the PSID stabilized IPT weights

Again, we used multiple imputation with multilevel models (20 multiple imputed data-sets). When the county of residence was missing during the exposure period, we employed LOCF and NOCB methods. About $44 \%$ of the PSID sample moved to a different county during the exposure time. On average, matched counties with the HIPD data cover 17.3 out of 20 years of exposure, and only $1.4 \%$ of the whole sample report less than seven years of exposure.

Lastly, we used different outcome models depending on the nature of the dependent variable: ordinal logistic regression when estimating the effect of income mobility and inequality on selfreported health status, Generalized linear models (GLM) for BMI and depression symptoms, logistic regression in the case current smoking status, and quasi-Poisson models for the number of cigarettes smoked during the last month. The outcome models adjusted only for baseline and time-invariant covariates, and considered sampling design variables (strata, clusters) and weights.

\footnotetext{
${ }^{16}$ The PSID screens mood or anxiety disorder using the Kessler Psychological Distress Scale (K6) in 2001-2003, 2007-2017. The scale includes six items: During the past 30 days, about how often did you feel nervous, hopeless, restless or fidgety, so depressed that nothing could cheer you up, that everything was an effort, worthless.
} 


\section{Results}

\subsection{Sample characteristics}

Tables S5 and S6 in the Methodological Supplement show descriptive statistics of our analytical samples and the proportion of missing data by variable. These tables provide insights about differences regarding design, composition, and length of exposure in NLSY97 and PSID samples. For instance, among NLSY97 respondents, the first interview was, on average, at age 14 (min 12, max 18), while the last interview was at age 33. This contrasts with the PSID sample whose respondents entered the study since they were born, and had their last interview at age 37 on average (min 30, max 47). Due to these differences in measurement, the number of residential changes is also different between samples. Whereas the proportion of NLSY97 respondents who moved to another county during the observation period was $27 \%, 44 \%$ of PSID interviewees have changed their residential county in 20 years.

In addition to these differences, Tables $[55$ and $\mathrm{S} 6$ show the PSID sample has slightly more White respondents than the NLSY97 (59\% versus 52\%). However, on average, the PSID respondents seem to have lived in counties with a higher proportion of African-Americans (19\% versus 15\% among NLSY97 respondents). The nature of some covariates also differ. For example, while parents' education is time-invariant in NLSY97 (only measured at the baseline), the PSID recorded that variable over time (i.e., time-variant).

The outcome variables - measured at the end of the follow-up period - show relatively similar values in both samples, except for current smoking. The self-reported health scale (1-5) is around 3.5 points in both samples, while the BMI ranges between 28.7 and 28.9, and the proportion of respondents currently smoking is higher in the NLSY97 (30\%) than in the PSID (20\%). The remaining outcome variables (depressive symptoms and smoking intensity) are not strictly comparable. The proportion of missing data in the outcome variables ranges between $20 \%$ and $31 \%$, and reaches its maximum in BMI (31\% in the PSID, and 24\% in the NLSY97).

For the rest of the covariates, missing data are considerably lower in the PSID than in the 
NLSY97. While the PSID's highest proportion of missing cases is observed in the variable weight of the respondent when was born (12\%), the NLSY97 has considerably higher levels of missing data, especially in time-variant variables such as household income (69\%), family size (35\%), and parents' working status (32\%). This pattern is due, in part, to the design of the NLSY97 study. As the observation window did not always start at age 12 , when the first interview was after age 12 , no information was collected on several covariates between age 12 and the age of the first interview. In those cases, we had to use retrospective parents' reports to determine where respondents lived when they were 12 years old and imputed missing covariates during that period.

We note that, although we compare the results from these two datasets, the descriptive tables S5 and S6 show relevant differences in design, composition, and exposure in the NLSY97 and PSID analytical samples that need be considered when interpreting our findings.

\subsection{IPT Weights}

Tables S7, S8, S11 and S14 in the Methodological Supplement show descriptive statistics of the stabilized IPT weights separated by sample and type of exposure variable: income mobility or income inequality, continuous or categorical, residualized or non-residualized. These descriptives come from IPT weights estimated using 20 different datasets with imputed data, and linear or ordinal logistic regression depending on the nature of the exposure variables (continuous or categorical). Because we adjusted attrition through imputation, we did not compute attrition weights.

IPT weights exhibit desirable properties when observed means are close to one, and they have small variance. Tables S7, S8, S11 and S14 show that all estimated weights are well-behaved and centered around one (ranging from 0.98 to 1.08). We found, however, substantial differences regarding the variability of the IPT weights. First, as expected, the variability of weights was much higher when using a continuous exposure treatment than a categorical one (quintile). Second, very high standard deviations of weights were mostly due to outliers. For instance, Tables $\mathrm{S} 11$ and $\mathbf{S 1 4}$ show that the standard deviation of the continuous version of the Gini coefficient and upward mobility are considerably large. However, once weights are truncated at the $1^{\text {th }}$ and $99^{\text {th }}$ percentiles, 
weights become stable, and standard deviations decrease considerably. Thus, we decided to use truncated weights in order to improve the efficiency of estimates and avoid the disproportionate influence of extreme observations (Hernán \& Robins, 2006; van der Wal \& Geskus, 2011; Thoemmes \& Ong, 2016).

\subsection{NLYS97 estimates}

We estimated four sets of models based on the following categories: unadjusted or adjusted, continuous or categorical exposure. Unadjusted models provide naive estimates by regressing exposure on outcomes without adjustments and IPT weighting, except for sampling weighting. Within each set of models, we ran independent models for each exposure variable: relative income mobility, absolute income mobility, and income inequality.

Table 1 shows the coefficients of average residualized exposure on health outcomes for NLSY97. The first three rows in Table 1 show the naive association of average county exposure from age 12 to 20 with five health outcomes. All the exposure treatments represent a negative trait, so we expect adverse consequences for health. To keep consistency with that interpretation, we multiplied upward mobility by -1 , so that any increase in exposure would consist of a negative condition (i.e., less income mobility, more rigidity of the stratification system, more inequality).

The rank-rank score coefficient (relative income mobility) for self-reported health status is 0.02 $(\mathrm{SE}=0.04)$. Because that coefficient comes from an ordinal logistic regression, an increase in one standard deviation on the average exposure to a rigid stratification enviroment implies an increment of $2 \%(\exp (0.02)=1.02)$ in the odds of reporting excellent health (versus bad health) ${ }^{17}$ Similar coefficients are observed regarding upward mobility (i.e., absolute income mobility) and income inequality (Gini coefficient). However, those estimates are very imprecise and noisy. Coefficients regarding BMI and depression symptoms in Table 1 are easier to interpret. An increase of one standard deviation in the rank-rank score rises BMI by 0.04 points, and the depression scale by

\footnotetext{
${ }^{17}$ The proportional odds assumption in original logistic models is not simply that the odds are the same, but that odds ratios are the same across categories.
} 
0.02 points. Again, these estimates are very imprecise and switch their sign in a non-systematic fashion.

Smoking models show more systematic associations. Both increases in the rank-rank correlation or lack of upward mobility raise the odds ratio of smoking later in life by $25 \%$ and $23 \%$, respectively $\left(\beta_{\mathrm{rank}}=0.22, \mathrm{SE}_{\mathrm{rank}}=0.04, \beta_{\text {upward }}=0.21, \mathrm{SE}_{\text {upward }}=0.05\right)$. Surprisingly, the naive association between the Gini coefficient and current smoking is negative. Similar associations are observed when modeling the number of days smoking during the last day. The quasi-Poisson coefficients suggest increasing the rank-rank correlation or reducing upward mobility raise the incidence rate ratio by $23 \%$ and $22 \%$. Again, and contrary to our expectations, the naive Gini coefficient suggests a negative relationship between income inequality and days smoked during the last month.

In sum, unadjusted point estimates for income mobility were relatively small and not systematic across health outcomes. Only smoking behavior seems to have a systematic association with exposure to income mobility in the expected direction (the higher the income mobility, the better the health outcome). Depression, self-report health status, and BMI estimates, instead, are small and uncertain. As a sensitivity analysis, we estimated unadjusted models using non-residualized exposure variables. In that case, the association between exposure and health outcomes can be spurious as income mobility and inequality might relate to counties' characteristics that also impact health. Table S9 in the Methodological Supplement shows unadjusted models with non-residualized exposure variables. The patterns are relatively similar to the residualized exposure variable models. Most of the systematic associations are observed between income mobility and smoking, but also BMI.

The estimates discussed above might be biased because of non-random selection into counties (residential mobility) and confounding. The next set of results comes from models using a weighted pseudo-population in which county exposure at each wave is independent of prior timevarying covariates. The second section of Table 1 (adjusted models) shows IPT-weighted estimates of the effect of standardized income mobility and inequality exposure on health outcomes. Under 
assumptions of no unmeasured confounders, no model misspecification, and positivity - there is a non-zero probability of treatment for every level and combination of confounders (Hernán \& Robins, 2006) - stabilized IPT weighting provides unbiased estimates of average causal effects.

Most of the estimates are small and very imprecise to claim they are systematically positive or negative. Relative income mobility estimates on smoking and BMI seem slightly more precise, even after IPT weighting reduces them in about half with respect to the unadjusted estimates. We should note, though, that the standard errors in Table 1 are underestimated as we are using several outcomes and making multiple comparisons. Consequently, there is a higher chance of finding false positives. Moreover, given the small and noisy BMI estimate in the unadjusted model, the positive coefficient $\left(\beta_{\mathrm{BMI}}=0.33, \mathrm{SE}=0.16\right)$ in the adjusted model, still imprecise, must be interpreted with caution.

Finally, IPT weights using continuous exposure are sensitive and unstable due to parametric misspecification and outliers. Thus, we also estimated the effect of income mobility and inequality using a categorical version of exposure (quintile). Table 2 shows both unadjusted and adjusted models by health outcome. Although the pattern of the coefficients is similar to Table 1 , estimates tend to be - as expected - more precise and smaller due to the change in the scale of exposure (1 to 5). In some cases, coefficients even switch their sign. For instance, the unadjusted smoking coefficients for upward mobility and depression symptoms were, as expected, positive, but they become negative when using the categorical version of income upward mobility. Similar to Table 1. smoking estimates and BMI reveal a systematic relationship with relative income mobility. For instance, exposure to the most rigid stratification level (5) compared to counties in the $3^{\text {rd }}$ quintile (average), increases the odds of smoking by about $17 \%(\exp ((5-3) \times 0.08)=1.17)$. Again, although BMI has a positive and relatively precise coefficient, it only appears in the adjusted models what suggests it is not systematic 18

\footnotetext{
${ }^{18}$ Table $\mathrm{S} 10$ in the Methodological Supplement shows unadjusted models with non-residualized and categorical exposure variables. They show a similar pattern to the models already discussed.
} 


\subsection{PSID estimates}

The same set of models was estimated with the PSID sample. Although the period of exposure in this case is longer (from birth to age 20), the sample size of the 1975-1980 cohort is substantially smaller (2,273 respondents versus 8,810 in the NLSY97). Table 3 shows unadjusted and adjusted models for residualized income mobility and inequality on health outcomes. In contrast to the NLSY97 results, most of the non-adjusted coefficients are very imprecise and noisy. The only stable coefficients are those related to current smoking and number of cigarettes. The adjusted results in the bottom section of Table 3, in turn, show effects that, although most of the time in the expected direction, are so imprecise to suggest either positive or negative consequences for health. 19 Using a categorical version of exposure do not change results or improve estimates (see Table 4). Similar to the NLSY97 analysis, we estimated the model using non-residualized exposure variables to examine how sensitive our results were to aggregate adjustments of income inequality and mobility indexes. Tables S12 and S13 in the Methodological Supplement show the effects of exposure to a rigid or unequal stratification environment are non-systematic and noisy, even in the non-adjusted models.

\section{Conclusion and discussion}

This paper aims to estimate the effect of average exposure during childhood and adolescence to a rigid and unequal stratification environment on health outcomes and behaviors during adulthood. Thus, we extend previous research on the association between income mobility, health, and mortality, and assess the hypothesis that early-life exposure to a given income mobility regime may determine health later and explain, at least in part, the longevity gaps observed across places in the U.S.

Our analysis suggests the connection between income mobility and health is not as systematic as previous research shows. Our most robust effects are related to smoking behavior (i.e.,

\footnotetext{
${ }^{19}$ Similar results were obtained when redefining the cohort of respondents (e.g., those born between 1970 and 1985 ) in order to increase the sample size, at the cost of adding imprecision to the exposure measures.
} 
currently smoking, number of days smoked in the last month), although only for NLSY97 and relative income mobility (rank-rank correlation). This particular finding implies income mobility might directly affect behaviors critically associated with modern chronic illnesses, such as smoking uptake and desistance, alcohol consumption, substance abuse, choices of diet and physical activity, as theory indicates. However, we were not able to replicate these findings using the PSID sample that comprehensively measures exposure from birth to age 20 , and where selection bias could be better reduced by using IPT weights. Surprisingly, not even our naive coefficients - without adjustments - were strong or systematic enough. The association between income inequality and health outcomes did not hold, either.

The relative consistency between unadjusted and adjust models suggests selection is not the only reason there might be inconsistencies between previous aggregate and cross-sectional results and our findings. Although adjustment does reduce the NLSY97 smoking coefficients, unadjusted models do not seem reveal a systematic association between average exposure to economic opportunities and health outcomes as previous research shows (Venkataramani et al., 2016; Venkataramani et al., 2015; Venkataramani et al., 2020). There might be two explanations for these findings.

First, our results might be affected by measurement error. As recently Mogstad et al. (2020) have pointed out, income mobility measures and rankings computed by Chetty et al. (2014)'s and colleagues are estimates rather than true values, so they might carry considerable uncertainty as population size varies considerably across counties. Figure S1 in the Methodological Supplement provides some evidence on how measurement error might affect our results. Figure S1 shows most of the counties in the NLSY97 sample have a larger population than those counties excluded. This is expected given the usual sampling design of nationally representative samples. Given the population size of counties, we would expect higher uncertainty of estimates in the small counties. Figure $\mathrm{S} 1$ shows smaller counties do actually have the most extreme income mobility values, likely due to higher uncertainty. This seems to be especially the case of absolute income mobility (the correlation between population and upward mobility is around -0.37), although relative income mobility has also the most extreme values among smaller counties except that they are 
evenly distributed across positive and negative values. Thus, by mostly including bigger counties, the NLSY97 and PSID sampling scheme is excluding uncertain income mobility estimates from smaller counties. We agree that this is far from an optimal strategy to account for measurement error when estimating the effect of the county economic opportunity environment on health, but unfortunately, we do not have access to the standard errors of the income mobility estimates at the county level.

In comparison, Venkataramani et al. (2016)'s pool several cross-sectional samples from the Behavioral Risk Factor Surveillance Survey (BRFSS), reaching nearly 147,000 individuals of ages 25 to 35 . That sample covers 2242 counties, which represents about $78 \%$ of all the counties used by Chetty et al. (2014) to estimate income mobility. The same is true concerning aggregate analyses by Venkataramani et al. (2015) and Daza and Palloni (2018), where almost all the counties used by Chetty et al. (2014) were included in the analysis. In contrast, the NLSY97 and PSID samples cover only $55 \%$ and $39 \%$ of the counties, respectively. Although we do not know what would happen to previous research results if only the counties of NLSY97 and PSID samples were considered, or better, if the measurement error were considered in the analysis, our findings and the work by Mogstad et al. (2020) suggest that measurement error might exaggerate estimates. Future research will be needed to assess the consequences of measurement error thoroughly, provided the standard errors of income mobility estimates are available.

A second way to interpret our findings stresses the complexity of the effects being estimated. The mechanisms we outlined when discussing the reasons why we should expect a causal relationship between the stratification system and health are not simple. The size of these effects is probably small, and they might be relevant to specific groups of the population and not others (i.e., heterogeneity). For instance, an individual's family mobility experience might be equally influential and may offset harmful effects stemming from a place's income mobility. In this context, estimating the population's average effect would not necessarily provide an unbiased and robust estimate. We are also assuming - due to data limitations - that the aggregate level that matters is counties, but it perfectly possible that the adequate level when estimating these exposure effects 
is neighborhoods. These factors, in addition to measurement error, make it difficult to estimate long-term implications of society's opportunity system for health. Our results do not necessarily indicate the causal link between income mobility and health does not exist, but that our data and analytical strategies are not strong enough to show they are systematic and in the expected direction.

Some additional limitations of our specific analysis should be noted. First, although IPTweighted estimates avoid some problems associated with conditioning on observed time-varying confounders, selection bias may still occur if unobserved factors simultaneously affect decisions about where to live and health behavior. Unfortunately, the assumption of no unobserved confounding is not testable with observed data, despite adjusting for an extensive set of observed covariates. Second, although we use different specifications and results were relatively stable (not shown), treatment models may still be misspecified. We also need to assume a positive probability of treatment for every level and combination of prior confounders. Theoretically, there is no reason to expect zero treatment probabilities across a set of covariates over time subgroups, except for the inherent limitations of sampling. Third, we assume that measuring income mobility in a cohort a measure that necessarily realizes in the future - accounts for the latent socioeconomic rigidity to which people were exposed early in life. It is possible that what really matters is the income mobility of the previous generation, as those experiences would determine the socialization and investments of the next generation. Finally, we imputed missing values and adjusted attrition using multiple imputation. Even though we obtained reasonable values and distributions, it is still possible that our imputation models are misspecified, and assumptions such as missing at random (MAR) do not hold.

Overall, our paper is the first to provide individual estimates of the effect of income mobility on health using a precise definition of exposure and accounting for selection and time-varying confounders. Thus, by focusing on individual outcomes during adulthood and influences during early formative years, we assess more directly the hypothesis that growing up in a community with a rigid stratification system may discourage adoption of behaviors that provide immediate rewards 
but are highly noxious, difficult to abandon, and bearers of large effects on health that take a long time to manifest. Our results suggest a weak connection between income mobility and health, and a non-systematic link between income inequality and health outcomes, even using two different datasets. Selection and measurement error might exaggerate estimates from previous research, but it is also possible that income mobility effects are relatively small and heterogeneous, making it difficult to estimate them precisely. Future research should focus on finding new indicators of socioeconomic mobility, both at the individual and appropriate aggregate level, to assess the magnitude of the consequences of the society system of opportunities for health. 


\section{Tables and Figures}

Table 1: Estimates of average residualized continuous exposure on health indicators, NLSY97

\begin{tabular}{|c|c|c|c|c|c|}
\hline & Health status & BMI & Depression & Smoking & Days smoking last month \\
\hline \multicolumn{6}{|l|}{ Unadjusted models } \\
\hline \multirow[t]{2}{*}{ Rank-rank } & 0.02 & 0.04 & $0.02^{*}$ & $0.22^{* * *}$ & $0.21^{* * *}$ \\
\hline & $(0.04)$ & $(0.13)$ & $(0.01)$ & $(0.04)$ & $(0.04)$ \\
\hline \multirow[t]{2}{*}{ Upward mobility $\times-1$} & 0.04 & -0.04 & $0.02^{*}$ & $0.21^{* * *}$ & $0.20^{* * *}$ \\
\hline & $(0.04)$ & $(0.16)$ & $(0.01)$ & $(0.05)$ & $(0.05)$ \\
\hline \multirow[t]{2}{*}{ Gini } & 0.01 & -0.08 & -0.00 & $-0.15^{* * *}$ & $-0.16^{* * *}$ \\
\hline & $(0.03)$ & $(0.11)$ & $(0.01)$ & $(0.03)$ & $(0.03)$ \\
\hline \multicolumn{6}{|l|}{ Adjusted models } \\
\hline \multirow[t]{2}{*}{ Rank-rank } & -0.03 & $0.33^{*}$ & 0.01 & $0.12^{* *}$ & $0.11^{* *}$ \\
\hline & $(0.04)$ & $(0.16)$ & $(0.01)$ & $(0.05)$ & $(0.04)$ \\
\hline \multirow[t]{2}{*}{ Upward mobility $\times-1$} & 0.02 & 0.11 & 0.01 & 0.07 & 0.05 \\
\hline & $(0.05)$ & $(0.15)$ & $(0.01)$ & $(0.05)$ & $(0.04)$ \\
\hline \multirow[t]{2}{*}{ Gini } & 0.02 & -0.07 & -0.01 & -0.05 & $-0.07^{*}$ \\
\hline & $(0.04)$ & $(0.11)$ & $(0.01)$ & $(0.04)$ & $(0.03)$ \\
\hline Individuals & 8810 & 8810 & 8810 & 8810 & 8810 \\
\hline
\end{tabular}

Each coefficient represents a model. Coefficients and standard errors are combined estimates from 20 multiple imputed datasets. Analyses based on exposure from 12 to 20 years old. We estimate different models depending on the outcome: Ordinal regression (selfreported health), General linear model (BMI, depression), Logistic regression (smoking), Quasi-Poisson regression (days smoking last month). ${ }^{* * *} p<0.001,{ }^{* *} p<0.01,{ }^{*} p<0.05$ 
Table 2: Estimates of average residualized categorical (quintile) exposure on health indicators, NLSY97

\begin{tabular}{|c|c|c|c|c|c|}
\hline & Health status & BMI & Depression & Smoking & Days smoking last month \\
\hline \multicolumn{6}{|l|}{ Unadjusted models } \\
\hline \multirow[t]{2}{*}{ Rank-rank } & 0.01 & 0.07 & 0.01 & $0.11^{* * *}$ & $0.11^{* * *}$ \\
\hline & $(0.02)$ & $(0.07)$ & $(0.01)$ & $(0.02)$ & $(0.02)$ \\
\hline \multirow[t]{2}{*}{ Upward mobility $\times-1$} & -0.01 & 0.00 & $-0.01^{*}$ & $-0.08^{* * *}$ & $-0.08^{* * *}$ \\
\hline & $(0.02)$ & $(0.08)$ & $(0.01)$ & $(0.02)$ & $(0.02)$ \\
\hline \multirow[t]{2}{*}{ Gini } & 0.02 & -0.04 & -0.00 & $-0.10^{* * *}$ & $-0.10^{* * *}$ \\
\hline & $(0.02)$ & $(0.07)$ & $(0.00)$ & $(0.02)$ & $(0.02)$ \\
\hline \multicolumn{6}{|l|}{ Adjusted models } \\
\hline \multirow[t]{2}{*}{ Rank-rank } & -0.01 & $0.18^{*}$ & 0.00 & $0.08^{* * *}$ & $0.07^{* * *}$ \\
\hline & $(0.02)$ & $(0.07)$ & $(0.01)$ & $(0.02)$ & $(0.02)$ \\
\hline \multirow[t]{2}{*}{ Upward mobility $\times-1$} & -0.01 & -0.05 & -0.01 & -0.03 & -0.02 \\
\hline & $(0.02)$ & $(0.07)$ & $(0.01)$ & $(0.02)$ & $(0.02)$ \\
\hline \multirow[t]{2}{*}{ Gini } & 0.03 & -0.04 & -0.00 & -0.04 & $-0.05^{* *}$ \\
\hline & $(0.03)$ & $(0.08)$ & $(0.00)$ & $(0.03)$ & $(0.02)$ \\
\hline Individuals & 8810 & 8810 & 8810 & 8810 & 8810 \\
\hline
\end{tabular}

Each coefficient represents a model. Coefficients and standard errors are combined estimates from 20 multiple imputed datasets. Analyses based on exposure from 12 to 20 years old. We estimate different models depending on the outcome: Ordinal regression (selfreported health), General linear model (BMI, depression), Logistic regression (smoking), Quasi-Poisson regression (days smoking last month). ${ }^{* * *} p<0.001,{ }^{* *} p<0.01,{ }^{*} p<0.05$ 
Table 3: Estimates of average residualized continuous exposure on health indicators, PSID

\begin{tabular}{|c|c|c|c|c|c|}
\hline & Health status & BMI & Depression & Smoking & Cigarettes smoked \\
\hline \multicolumn{6}{|l|}{ Unadjusted models } \\
\hline \multirow[t]{2}{*}{ Rank-rank } & -0.03 & -0.10 & 0.03 & $0.26^{*}$ & $0.26^{*}$ \\
\hline & $(0.08)$ & $(0.32)$ & $(0.03)$ & $(0.13)$ & $(0.13)$ \\
\hline \multirow[t]{2}{*}{ Upward mobility $\times-1$} & -0.10 & 0.40 & 0.04 & 0.31 & $0.37^{*}$ \\
\hline & $(0.10)$ & $(0.36)$ & $(0.04)$ & $(0.20)$ & $(0.18)$ \\
\hline \multirow[t]{2}{*}{ Gini } & 0.11 & -0.34 & -0.01 & -0.15 & $-0.16^{*}$ \\
\hline & $(0.08)$ & $(0.29)$ & $(0.02)$ & $(0.09)$ & $(0.08)$ \\
\hline \multicolumn{6}{|l|}{ Adjusted models } \\
\hline \multirow[t]{2}{*}{ Rank-rank } & -0.01 & -0.15 & 0.03 & 0.10 & 0.18 \\
\hline & $(0.09)$ & $(0.26)$ & $(0.04)$ & $(0.15)$ & $(0.14)$ \\
\hline \multirow[t]{2}{*}{ Upward mobility $\times-1$} & 0.01 & 0.09 & 0.03 & 0.05 & 0.14 \\
\hline & $(0.10)$ & $(0.32)$ & $(0.04)$ & $(0.19)$ & $(0.17)$ \\
\hline \multirow[t]{2}{*}{ Gini } & 0.13 & -0.12 & -0.00 & -0.04 & -0.08 \\
\hline & $(0.09)$ & $(0.28)$ & $(0.02)$ & $(0.11)$ & $(0.08)$ \\
\hline Individuals & 2273 & 2273 & 2273 & 2273 & 2273 \\
\hline
\end{tabular}

Each coefficient represents a model. Coefficients and standard errors are combined estimates from 20 multiple imputed datasets. Analyses based on exposure from 1 to 20 years old. We estimate different models depending on the outcome: Ordinal regression (self-reported health), General linear model (BMI, depression), Logistic regression (smoking), QuasiPoisson regression (cigarettes smoked). ${ }^{* *} p<0.001,{ }^{* *} p<0.01,{ }^{*} p<0.05$ 
Table 4: Estimates of average residualized categorical (quintile) exposure on health indicators, PSID

\begin{tabular}{|c|c|c|c|c|c|}
\hline & Health status & BMI & Depression & Smoking & Cigarettes smoked \\
\hline \multicolumn{6}{|l|}{ Unadjusted models } \\
\hline \multirow[t]{2}{*}{ Rank-rank } & -0.00 & -0.01 & 0.01 & 0.09 & 0.09 \\
\hline & $(0.05)$ & $(0.17)$ & $(0.02)$ & $(0.07)$ & $(0.07)$ \\
\hline \multirow[t]{2}{*}{ Upward mobility $\times-1$} & 0.04 & -0.34 & -0.01 & $-0.16^{*}$ & $-0.20^{* *}$ \\
\hline & $(0.05)$ & $(0.18)$ & $(0.02)$ & $(0.08)$ & $(0.07)$ \\
\hline \multirow[t]{2}{*}{ Gini } & 0.08 & $-0.32^{*}$ & -0.00 & -0.08 & -0.06 \\
\hline & $(0.05)$ & $(0.15)$ & $(0.01)$ & $(0.06)$ & $(0.05)$ \\
\hline \multicolumn{6}{|l|}{ Adjusted models } \\
\hline \multirow[t]{2}{*}{ Rank-rank } & 0.03 & -0.09 & 0.01 & 0.03 & 0.03 \\
\hline & $(0.05)$ & $(0.13)$ & $(0.02)$ & $(0.08)$ & $(0.07)$ \\
\hline \multirow[t]{2}{*}{ Upward mobility $\times-1$} & -0.01 & -0.13 & -0.01 & -0.06 & -0.10 \\
\hline & $(0.05)$ & $(0.18)$ & $(0.02)$ & $(0.08)$ & $(0.07)$ \\
\hline \multirow[t]{2}{*}{ Gini } & 0.09 & -0.23 & -0.00 & 0.00 & 0.00 \\
\hline & $(0.05)$ & $(0.14)$ & $(0.01)$ & $(0.06)$ & $(0.05)$ \\
\hline Individuals & 2273 & 2273 & 2273 & 2273 & 2273 \\
\hline
\end{tabular}

Each coefficient represents a model. Coefficients and standard errors are combined estimates from 20 multiple imputed datasets. Analyses based on exposure from 1 to 20 years old. We estimate different models depending on the outcome: Ordinal regression (self-reported health), General linear model (BMI, depression), Logistic regression (smoking), QuasiPoisson regression (cigarettes smoked). ${ }^{* * *} p<0.001,{ }^{* *} p<0.01,{ }^{*} p<0.05$ 


\section{References}

Blane, D., Harding, S., \& Rosato, M. (1999). Does social mobility affect the size of the socioeconomic mortality differential?: Evidence from the Office for National Statistics Longitudinal Study. Journal of the Royal Statistical Society: Series A (Statistics in Society), 162(1), 5970.

Blane, D., Smith, G. D., \& Bartley, M. (1993). Social selection: What does it contribute to social class differences in health? Sociology of Health \& Illness, 15(1), 1-15.

Case, A., \& Deaton, A. (2020). Deaths of Despair and the Future of Capitalism. Princeton, Princeton University Press.

Case, A., Lubotsky, D., \& Paxson, C. (2002). Economic Status and Health in Childhood: The Origins of the Gradient. The American Economic Review, 92(5), 1308-1334.

Case, A., \& Paxson, C. (2011). The Long Reach of Childhood Health and Circumstance: Evidence from the Whitehall II Study*. The Economic Journal, 121(554), F183-F204.

Chandola, T., Bartley, M., Sacker, A., Jenkinson, C., \& Marmot, M. (2003). Health selection in the Whitehall II study, UK. Social Science \& Medicine, 56(10), 2059-2072.

Chetty, R., Grusky, D., Hell, M., Hendren, N., Manduca, R., \& Narang, J. (2017). The fading American dream: Trends in absolute income mobility since 1940. Science, 356(6336), 398406.

Chetty, R., Hendren, N., Kline, P., \& Saez, E. (2014). Where is the land of Opportunity? The Geography of Intergenerational Mobility in the United States. The Quarterly Journal of Economics, 129(4), 1553-1623.

Chetty, R., Stepner, M., Abraham, S., Lin, S., Scuderi, B., Turner, N., Bergeron, A., \& Cutler, D. (2016). The Association Between Income and Life Expectancy in the United States, 2001-2014. JAMA, 315(16), 1750.

Cunha, F., \& Heckman, J. J. (2009). The Economics and Psychology of Inequality and Human Development. Journal of the European Economic Association, 7(2-3), 320-364. 
Daza, S., \& Palloni, A. (2018). Income Inequality, Social Mobility and Mortality in the U.S. CDE Working Paper, University of Wisconsin-Madison, (02).

Dugoff, E. H., Schuler, M., \& Stuart, E. A. (2014). Generalizing observational study results: Applying propensity score methods to complex surveys. Health Services Research, 49(1), 284303.

Eigsti, I.-M., Zayas, V., Mischel, W., Shoda, Y., Ayduk, O., Dadlani, M. B., Davidson, M. C., Aber, J. L., \& Casey, B. J. (2006). Predicting Cognitive Control From Preschool to Late Adolescence and Young Adulthood. Psychological Science, 17(6), 478-484.

Fox, A. J., Goldblatt, P. O., \& Adelstein, A. M. (1982). Selection and mortality differentials. Journal of Epidemiology \& Community Health, 36(2), 69-79.

Grossman, M. (1972). On the Concept of Health Capital and the Demand for Health. Journal of Political Economy, 80(2), 223-255.

Grossman, M. (2000). Chapter 7 - The Human Capital Model* (A. J. C. a. J. P. Newhouse, Ed.). In A. J. C. a. J. P. Newhouse (Ed.), Handbook of Health Economics. Elsevier.

Haushofer, J., \& Fehr, E. (2014). On the psychology of poverty. Science, 344(6186), 862-867.

Heckman, J. J. (2007). The Economics, Technology and Neuroscience of Human Capability Formation. Proceedings of the National Academy of Sciences, 104(33), 13250-13255.

Hernán, M. A., Brumback, B. A., \& Robins, J. M. (2002). Estimating the causal effect of zidovudine on CD4 count with a marginal structural model for repeated measures. Statistics in medicine, 21(12), 1689-1709.

Hernán, M. A., \& Robins, J. M. (2006). Estimating causal effects from epidemiological data. Journal of Epidemiology and Community Health (1979-), 60(7), 578-586.

Hernán, M. Á., Brumback, B., \& Robins, J. M. (2000). Marginal structural models to estimate the causal effect of zidovudine on the survival of HIV-positive men. Epidemiology, 11(5), $561-570$.

Illsley, R. (1955). Social Class Selection and Class Differences in Relation to Stillbirths and Infant Deaths. British Medical Journal, 2(4955), 1520-1524. 
Knudsen, E. I., Heckman, J. J., Cameron, J. L., \& Shonkoff, J. P. (2006). Economic, neurobiological, and behavioral perspectives on building America's future workforce. Proceedings of the National Academy of Sciences, 103(27), 10155-10162.

Lynch, J., Smith, G. D., Harper, S. A., Hillemeier, M., Ross, N., Kaplan, G. A., \& Wolfson, M. (2004). Is income inequality a determinant of population health? Part 1. A systematic review. Milbank Quarterly, 82(1), 5-99.

Marmot, M. (2004). The status syndrome: How social standing affects our health and longevity. 2004. London: Bloomsbury.

Mogstad, M., Romano, J. P., Shaikh, A., \& Wilhelm, D. (2020). Inference for Ranks with Applications to Mobility across Neighborhoods and Academic Achievement across Countries (Working Paper No. 26883). National Bureau of Economic Research.

Naimi, A. I., Moodie, E. E., Auger, N., \& Kaufman, J. S. (2014). Constructing Inverse Probability Weights for Continuous Exposures: A Comparison of Methods. Epidemiology, 25(2), 292299.

van Buuren, S. (2018). Flexible Imputation of Missing Data (Second Edition). Chapman and Hall/CRC.

van der Wal, W. M., \& Geskus, R. B. (2011). Ipw: An R package for inverse probability weighting. Journal of Statistical Software, 43(13), 1-23.

Palloni, A., Milesi, C., White, R. G., \& Turner, A. (2009). Early childhood health, reproduction of economic inequalities and the persistence of health and mortality differentials. Social Science \& Medicine, 68(9), 1574-1582.

Sampson, R. J., Morenoff, J. D., \& Gannon-Rowley, T. (2002). Assessing "Neighborhood Effects": Social Processes and New Directions in Research. Annual Review of Sociology, 28(1), 443478.

Sapolsky, R. M. (2005). The influence of social hierarchy on primate health. Science, 308(5722), 648-652. 
Schlam, T. R., Wilson, N. L., Shoda, Y., Mischel, W., \& Ayduk, O. (2013). Preschoolers' delay of gratification predicts their body mass 30 years later. The Journal of pediatrics, 162(1), 90-93.

Shonkoff, J. P., Boyce, W. T., \& McEwen, B. S. (2009). Neuroscience, molecular biology, and the childhood roots of health disparities: Building a new framework for health promotion and disease prevention. JAMA, 301(21), 2252-9.

Solon, G. R. (1992). Intergenerational Income Mobility in the United States. The American Economic Review, 82(3), 393-408.

Thoemmes, F., \& Ong, A. D. (2016). A Primer on Inverse Probability of Treatment Weighting and Marginal Structural Models. Emerging Adulthood, 4(1), 40-59.

Venkataramani, A. S., Brigell, R., O’Brien, R., Chatterjee, P., Kawachi, I., \& Tsai, A. C. (2016). Economic opportunity, health behaviours, and health outcomes in the USA: A populationbased cross-sectional study. The Lancet Public Health, 1(1), e18-e25.

Venkataramani, A. S., Chatterjee, P., Kawachi, I., \& Tsai, A. C. (2015). Economic Opportunity, Health Behaviors, and Mortality in the United States. American Journal of Public Health, 106(3), 478-484.

Venkataramani, A. S., Daza, S., \& Ezekiel, E. (2020). Association of Social Mobility with the Income-Based Longevity Gap in the United States. A Cross-Sectional, County-Level Study. JAMA Internal Medicine, 180(3), 429-436.

Wodtke, G. T., Elwert, F., \& Harding, D. J. (2016). Neighborhood Effect Heterogeneity by Family Income and Developmental Period. American Journal of Sociology, 121(4), 1168-1222.

Wodtke, G. T., Harding, D. J., \& Elwert, F. (2011). Neighborhood Effects in Temporal Perspective The Impact of Long-Term Exposure to Concentrated Disadvantage on High School Graduation. American Sociological Review, 76(5), 713-736. 


\section{Methodological Supplement}

The code to create analytic data sets, models and plots is available at: https://github.com/sdaza/ dissertation/tree/master/ch03. Some of the variables in the paper are restricted and obtained under special contractual arrangements to protect the anonymity of respondents. These data are not available from the authors. Those interested in obtaining PSID restricted data should contact PSIDHelp@isr.umich.edu. Those interested in the NLSY restricted data, visit www.bls.gov/nls/ geocodeapp.htm.

\subsection{County coverage}

Figure $[$ 1 displays counties by the log of population and measures of income mobility and inequality, highlighting in red the counties included in the NLSY sample.

Figure S1: County income mobility and inequality over population by NLSY97 sample coverage

(a) NLSY97 Relative mobility

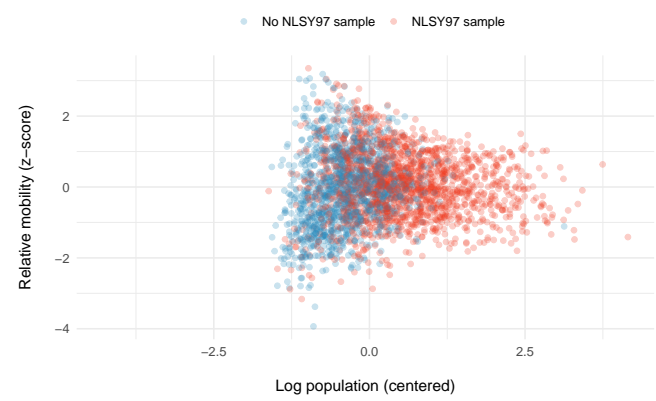

(b) NLSY97 Absolute mobility

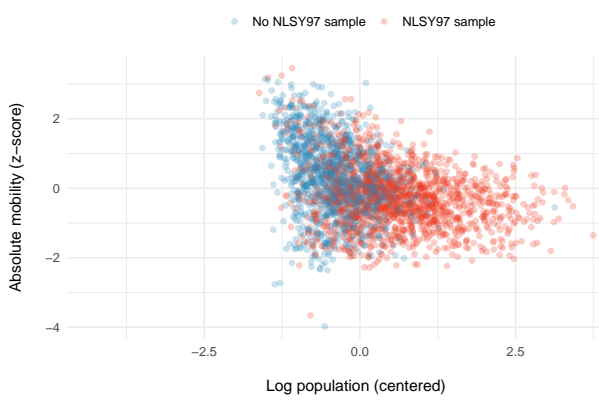

(c) NLSY97 Inequality

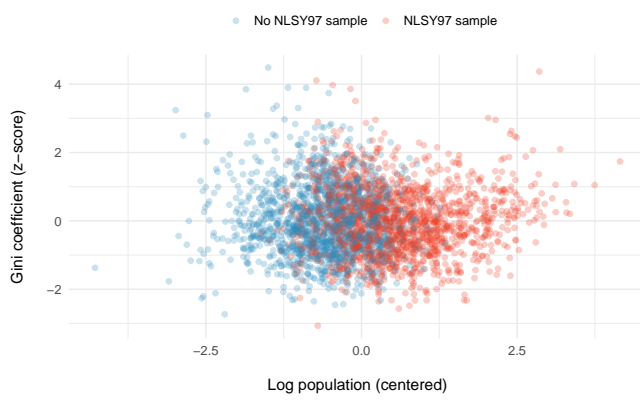


This figure provides insights on county coverage of the NLSY97 individual sample, and the relationship between the size of counties and the values of income mobility and inequality. Due to disclousure rules for restricted data, we cannot publish the same plots for the PSID sample. However, the patterns displayed in Figure $\mathrm{S} 1$ are similar to what we observed in the PSID sample.

\subsection{Descriptive statistics}

Tables S5 and S6 show descriptive statistics of the variables in our analysis. The PSID table cannot show min and max values due to disclosure rules for restricted data. 
Table S5: NLSY97 descriptive statistics of covariates and outcomes

\begin{tabular}{|c|c|c|c|c|c|c|}
\hline & Mean & SD & Min & Max & $\%$ Missing & Valid observations \\
\hline \multicolumn{7}{|l|}{ Time-invariant covariates } \\
\hline Male & 0.51 & 0.50 & 0.00 & 1.00 & 0.00 & 8810 \\
\hline Age first interview & 14.35 & 1.49 & 12.00 & 18.00 & 0.00 & 8810 \\
\hline Age last interview & 32.88 & 1.45 & 30.00 & 36.00 & 0.00 & 8810 \\
\hline \multicolumn{7}{|l|}{ Race-Ethnicity } \\
\hline White & 0.52 & 0.50 & 0.00 & 1.00 & 0.00 & 8810 \\
\hline Black & 0.26 & 0.44 & 0.00 & 1.00 & 0.00 & 8810 \\
\hline Hispanic & 0.21 & 0.41 & 0.00 & 1.00 & 0.00 & 8810 \\
\hline Mixed & 0.01 & 0.10 & 0.00 & 1.00 & 0.00 & 8810 \\
\hline ASVAB Test Score & 45.38 & 29.17 & 0.00 & 100.00 & 0.21 & 8810 \\
\hline Parent's Education (years) & 13.15 & 3.06 & 1.00 & 20.00 & 0.07 & 8810 \\
\hline Mother's age at birth of respondent & 25.48 & 5.39 & 12.00 & 54.00 & 0.07 & 8810 \\
\hline Number of residential moves by age 12 & 3.17 & 2.75 & 1.00 & 40.00 & 0.13 & 8810 \\
\hline Proportion moved to a different county & 0.27 & 0.44 & 0.00 & 1.00 & 0.00 & 8810 \\
\hline \multicolumn{7}{|l|}{ Time-variant covariates } \\
\hline Family size & 4.26 & 1.65 & 1.00 & 17.00 & 0.35 & 70480 \\
\hline Respondent living with any parent & 0.82 & 0.39 & 0.00 & 1.00 & 0.29 & 70480 \\
\hline Parent is working & 0.89 & 0.31 & 0.00 & 1.00 & 0.32 & 70480 \\
\hline Parent is married & 0.65 & 0.48 & 0.00 & 1.00 & 0.31 & 70480 \\
\hline Log household income & -0.11 & 2.42 & -10.40 & 2.98 & 0.69 & 70480 \\
\hline County log income & 0.74 & 1.08 & -3.07 & 4.32 & 0.00 & 70480 \\
\hline County log population & 1.76 & 1.15 & -1.62 & 4.15 & 0.00 & 70480 \\
\hline County proportion Black & 0.15 & 0.16 & 0.00 & 0.80 & 0.00 & 70480 \\
\hline Cumulative number of county moves & 0.15 & 0.47 & 0.00 & 6.00 & 0.00 & 70480 \\
\hline \multicolumn{7}{|l|}{ Exposure variables } \\
\hline County rank-rank correlation (original) & 0.26 & 0.07 & 0.04 & 0.53 & 0.00 & 70480 \\
\hline Quintile county rank-rank correlation (original) & 2.88 & 1.37 & 1.00 & 5.00 & 0.00 & 70480 \\
\hline Residualized county rank-rank correlation & -0.29 & 0.76 & -3.21 & 2.50 & 0.00 & 70480 \\
\hline Quintile residualized county rank-rank correlation & 2.52 & 1.30 & 1.00 & 5.00 & 0.00 & 70480 \\
\hline County upward mobility (original) & 0.44 & 0.05 & 0.33 & 0.67 & 0.00 & 70480 \\
\hline Quintile county upward mobility (original) & 2.18 & 1.16 & 1.00 & 5.00 & 0.00 & 70480 \\
\hline Residualized county upward mobility & 0.30 & 0.64 & -2.66 & 2.34 & 0.00 & 70480 \\
\hline Quintile residualized county upward mobility & 3.59 & 1.33 & 1.00 & 5.00 & 0.00 & 70480 \\
\hline County Gini coefficient (original) & 0.45 & 0.04 & 0.34 & 0.60 & 0.00 & 70480 \\
\hline Quintile county Gini coefficient (original) & 3.45 & 1.38 & 1.00 & 5.00 & 0.00 & 70480 \\
\hline Residualized county Gini coefficient & 0.21 & 0.92 & -2.84 & 4.99 & 0.00 & 70480 \\
\hline Quintile Residualized county Gini coefficient & 3.39 & 1.45 & 1.00 & 5.00 & 0.00 & 70480 \\
\hline \multicolumn{7}{|l|}{ Outcomes } \\
\hline Self-reported health & 3.63 & 1.00 & 1.00 & 5.00 & 0.21 & 8810 \\
\hline BMI & 28.70 & 5.96 & 15.00 & 40.00 & 0.24 & 8810 \\
\hline Depressive symptoms & 1.83 & 0.50 & 1.00 & 4.00 & 0.21 & 8810 \\
\hline Current smoking & 0.31 & 0.46 & 0.00 & 1.00 & 0.22 & 8810 \\
\hline Days smoked & 6.21 & 11.60 & 0.00 & 30.00 & 0.22 & 8810 \\
\hline
\end{tabular}

Note: Statistics based on non-imputed data. $\mathrm{SD}=$ Standard deviation. Observations correspond to respondents in the case of time-invariant and outcome variables, and person-years ( $\mathrm{N}$ times exposure) for time-variant variables. Outcomes were measured in 2015. 
Table S6: PSID descriptive statistics of covariates and outcomes

\begin{tabular}{|c|c|c|c|c|}
\hline & Mean & SD & $\%$ Missing & Observations \\
\hline \multicolumn{5}{|l|}{ Time-invariant covariates } \\
\hline Male & 0.46 & 0.50 & 0.00 & 2273 \\
\hline Age last interview & 37.03 & 3.25 & 0.00 & 2273 \\
\hline Birth year & 1980.23 & 3.18 & 0.00 & 2273 \\
\hline \multicolumn{5}{|l|}{ Race-Ethnicity } \\
\hline White & 0.59 & 0.49 & 0.00 & 2273 \\
\hline Black & 0.39 & 0.49 & 0.00 & 2273 \\
\hline Other & 0.02 & 0.14 & 0.00 & 2273 \\
\hline Weighted less than $55 \mathrm{oz}$ & 0.07 & 0.26 & 0.12 & 2273 \\
\hline Mother marital status at birth & 0.76 & 0.43 & 0.04 & 2273 \\
\hline Mother's age at birth of respondent & 25.18 & 4.95 & 0.00 & 2273 \\
\hline Proportion moved to a different county & 0.44 & 0.50 & 0.00 & 2273 \\
\hline \multicolumn{5}{|l|}{ Time-variant covariates } \\
\hline Family size & 4.30 & 1.38 & 0.06 & 45460 \\
\hline Respondent living with any parent & 0.73 & 0.45 & 0.06 & 45460 \\
\hline Parent's years of education & 12.87 & 2.44 & 0.07 & 45460 \\
\hline Parent is working & 0.61 & 0.49 & 0.06 & 45460 \\
\hline Parent is married & 0.83 & 0.38 & 0.06 & 45460 \\
\hline Log household income & 0.09 & 1.13 & 0.06 & 45460 \\
\hline County log income & 0.64 & 1.03 & 0.00 & 45460 \\
\hline County log population & 1.57 & 1.14 & 0.00 & 45460 \\
\hline County proportion Black & 0.19 & 0.19 & 0.00 & 45460 \\
\hline Cumulative number of county moves & 0.55 & 1.07 & 0.00 & 45460 \\
\hline \multicolumn{5}{|l|}{ Exposure variables } \\
\hline County rank-rank correlation (original) & 0.28 & 0.07 & 0.00 & 45460 \\
\hline Quintile county rank-rank correlation (original) & 3.27 & 1.34 & 0.00 & 45460 \\
\hline Residualized county rank-rank correlation & -0.20 & 0.73 & 0.00 & 45460 \\
\hline Quintile residualized county rank-rank correlation & 2.63 & 1.36 & 0.00 & 45460 \\
\hline County upward mobility (original) & 0.43 & 0.05 & 0.00 & 45460 \\
\hline Quintile county upward mobility (original) & 2.12 & 1.21 & 0.00 & 45460 \\
\hline Residualized county upward mobility & 0.25 & 0.59 & 0.00 & 45460 \\
\hline Quintile residualized county upward mobility & 3.56 & 1.28 & 0.00 & 45460 \\
\hline County Gini coefficient (original) & 0.45 & 0.04 & 0.00 & 45460 \\
\hline Quintile county Gini coefficient (original) & 3.46 & 1.39 & 0.00 & 45460 \\
\hline Residualized county Gini coefficient & 0.11 & 0.83 & 0.00 & 45460 \\
\hline Quintile Residualized county Gini coefficient & 3.23 & 1.40 & 0.00 & 45460 \\
\hline \multicolumn{5}{|l|}{ Outcomes } \\
\hline Self-reported health & 3.53 & 0.99 & 0.23 & 2273 \\
\hline BMI & 28.91 & 5.90 & 0.31 & 2273 \\
\hline Depressive symptoms & 1.63 & 0.67 & 0.23 & 2273 \\
\hline Current smoking & 0.20 & 0.40 & 0.23 & 2273 \\
\hline Number of cigarettes & 2.15 & 5.35 & 0.23 & 2273 \\
\hline
\end{tabular}

Note: Statistics based on non-imputed data. $\mathrm{SD}=$ Standard deviation. Observations correspond to respondents in the case of time-invariant and outcome variables, and person-years ( $\mathrm{N}$ times exposure) for time-variant variables. Outcomes were measured in 2017. 


\subsection{Imputation}

We employed multiple imputation for item non-response and attrition. For each exposure variable (e.g., relative and absolute income mobility), we ran multilevel models to impute values for both time-variant and invariant covariates. We produced 20 complete data-sets and pooled the results using Rubin's Rules (van Buuren, 2018).

Multiple imputation model specifications are available in our code repository. For each exposure variable, we produced 20 complete datasets per data source (PSID and NLSY9). Different imputed data-sets were created for residualized and non-residualized, continuous and categorical exposure (360 datasets in total). We assessed convergence and feasibility of results using the criteria suggested by van Buuren, 2018. For instance, Figure S3 and S4 show iteration plots of outcomes variables ( $\mathrm{a}$ and $\mathrm{b}$ ) and the comparison between observed and imputed distribution of outcomes ( $\mathrm{d}$ and $\mathrm{c})$. In general, convergence plots looked fine as they mix not systematically after 20 iterations. The distribution of outcomes also seemed reasonable, and no ill behavior of estimates was observed.

Figure S3: NLSY97 Imputation plots with relative mobility as exposure, 20 iterations

(a) Iterations

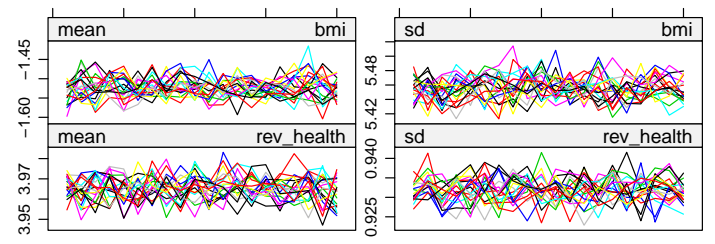

(b) Iterations

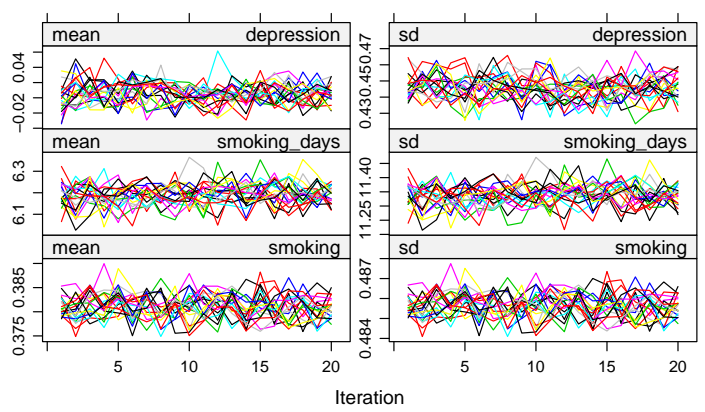


(c) Imputations vs Observed Values

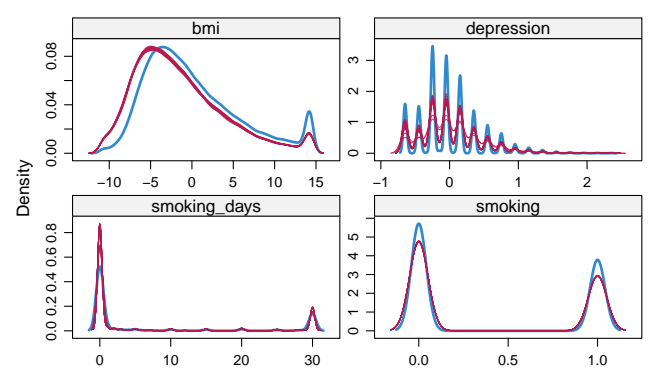

(d) Imputations vs Observed Values

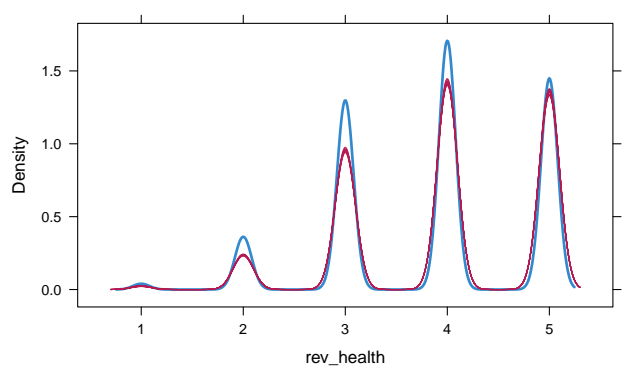


Figure S4: PSID Imputation plots with relative mobility as exposure, 20 iterations

(a) Iterations

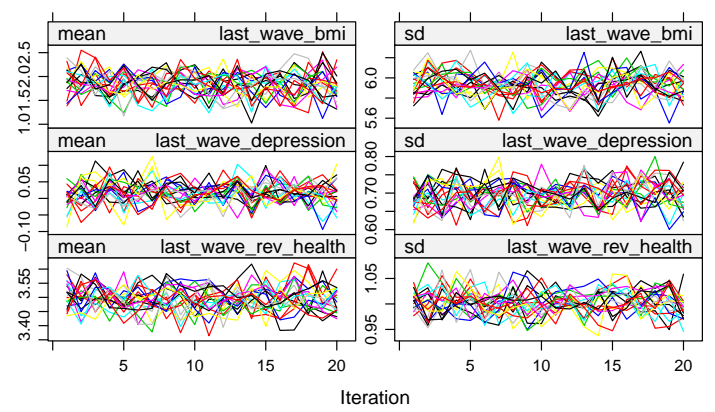

(c) Imputations vs Observed Values

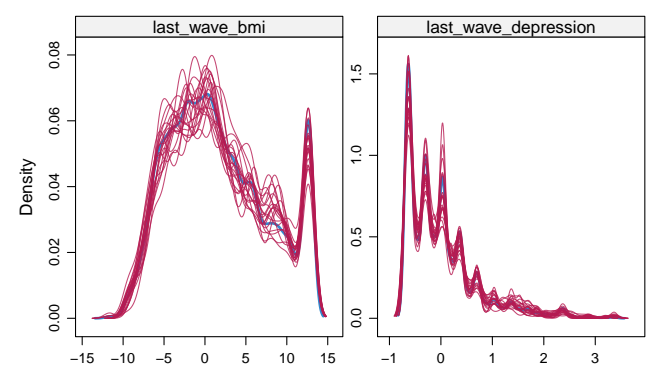

(b) Iterations

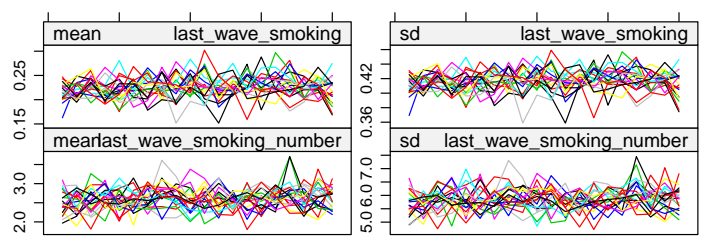

Iteration

(d) Imputations vs Observed Values

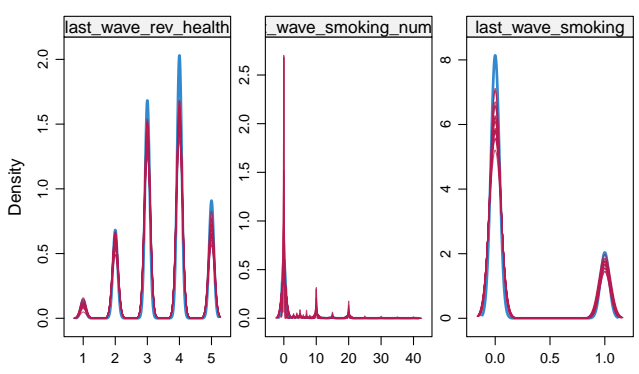




\subsection{IPT weights (residualized models)}

Table S7: NLSY97 Stabilized treatment weights (residualized)

\begin{tabular}{lcccccc}
\hline & & & \multicolumn{5}{c}{ Percentiles } \\
\cline { 5 - 7 } Weight & Mean & SD & 1st & 25th & 75th & 99 th \\
\hline Continuous exposure & & & & & & \\
Rank-rank & 1.02 & 0.72 & 0.28 & 0.81 & 1.09 & 3.07 \\
Upward mobility & 1.02 & 0.48 & 0.34 & 0.82 & 1.09 & 2.80 \\
Gini & 1.02 & 1.01 & 0.32 & 0.84 & 1.05 & 3.04 \\
Categorical (quintile) & exposure & & & & & \\
Rank-rank & 1.00 & 0.38 & 0.49 & 0.83 & 1.08 & 2.58 \\
Upward mobility & 1.00 & 0.34 & 0.54 & 0.82 & 1.10 & 2.27 \\
Gini & 1.00 & 0.41 & 0.45 & 0.80 & 1.10 & 2.56 \\
\hline
\end{tabular}

Analyses based on exposure from 12 to 20 years old. Statistics based on 20 multiple imputed datasets.

Table S8: PSID Stabilized treatment weights (residual exposure)

\begin{tabular}{lcccccc}
\hline & & & \multicolumn{5}{c}{ Percentiles } \\
\cline { 5 - 7 } Weight & Mean & SD & 1st & 25th & 75th & 99 th \\
\hline Continuous exposure & & & & & & \\
Rank-rank & 1.03 & 0.78 & 0.34 & 0.83 & 1.10 & 2.75 \\
Upward mobility & 1.03 & 0.90 & 0.45 & 0.87 & 1.08 & 2.11 \\
Gini & 1.00 & 0.28 & 0.43 & 0.89 & 1.06 & 2.00 \\
Categorical (quintile) exposure & & & & & \\
Rank-rank & 1.00 & 0.29 & 0.55 & 0.82 & 1.13 & 1.90 \\
Upward mobility & 1.00 & 0.25 & 0.59 & 0.86 & 1.10 & 1.73 \\
Gini & 1.00 & 0.24 & 0.53 & 0.88 & 1.09 & 1.81 \\
\hline
\end{tabular}

Analyses based on exposure from 1 to 20 years old. Statistics based on 20 multiple imputed datasets. 


\subsection{Non-residualized models}

For completeness, we show the results with non-residualized exposure treatments and IPT weight statistical descriptives for both the NLSY97 and PSID.

\subsubsection{NLSY97}

Table S9: Estimates of average continuous exposure on health indicators, NLSY97

\begin{tabular}{|c|c|c|c|c|c|}
\hline & Health status & BMI & Depression & Smoking & Days smoking last month \\
\hline \multicolumn{6}{|l|}{ Unadjusted models } \\
\hline \multirow[t]{2}{*}{ Rank-rank } & -0.04 & $0.39^{* * *}$ & 0.00 & $0.13^{* * *}$ & $0.15^{* * *}$ \\
\hline & $(0.03)$ & $(0.11)$ & $(0.01)$ & $(0.04)$ & $(0.03)$ \\
\hline \multirow[t]{2}{*}{ Upward mobility $\times-1$} & -0.04 & $0.30^{* *}$ & -0.00 & -0.03 & -0.02 \\
\hline & $(0.03)$ & $(0.11)$ & $(0.01)$ & $(0.04)$ & $(0.04)$ \\
\hline \multirow[t]{2}{*}{ Gini } & -0.02 & 0.16 & -0.01 & $-0.12^{* * *}$ & $-0.12^{* * *}$ \\
\hline & $(0.03)$ & $(0.09)$ & $(0.01)$ & $(0.03)$ & $(0.03)$ \\
\hline \multicolumn{6}{|l|}{ Adjusted models } \\
\hline \multirow[t]{2}{*}{ Rank-rank } & -0.03 & $0.28^{*}$ & 0.02 & $0.11^{*}$ & $0.12^{* * *}$ \\
\hline & $(0.03)$ & $(0.13)$ & $(0.01)$ & $(0.05)$ & $(0.04)$ \\
\hline \multirow[t]{2}{*}{ Upward mobility $\times-1$} & 0.04 & -0.04 & 0.01 & 0.06 & 0.04 \\
\hline & $(0.05)$ & $(0.13)$ & $(0.01)$ & $(0.05)$ & $(0.04)$ \\
\hline \multirow[t]{2}{*}{ Gini } & 0.04 & -0.13 & -0.00 & -0.05 & $-0.08^{*}$ \\
\hline & $(0.04)$ & $(0.12)$ & $(0.01)$ & $(0.05)$ & $(0.03)$ \\
\hline Individuals & 8810 & 8810 & 8810 & 8810 & 8810 \\
\hline
\end{tabular}

Each coefficient represents a model. Coefficients and standard errors are combined estimates from 20 multiple imputed datasets. Analyses based on exposure from 12 to 20 years old. We estimate different models depending on the outcome: Ordinal regression (self-reported health), General linear model (BMI, depression), Logistic regression (smoking), Quasi-Poisson regression (days smoking last month). ${ }^{* * *} p<0.001,{ }^{* *} p<0.01,{ }^{*} p<0.05$ 
Table S10: Estimates of average categorical (quintile) exposure on health indicators, NLSY97

\begin{tabular}{|c|c|c|c|c|c|}
\hline & Health status & BMI & Depression & Smoking & Days smoking last month \\
\hline \multicolumn{6}{|l|}{ Unadjusted models } \\
\hline \multirow[t]{2}{*}{ Rank-rank } & -0.02 & $0.25^{* * *}$ & 0.00 & $0.08^{* * *}$ & $0.10^{* * *}$ \\
\hline & $(0.02)$ & $(0.06)$ & $(0.01)$ & $(0.02)$ & $(0.02)$ \\
\hline \multirow[t]{2}{*}{ Upward mobility $\times-1$} & 0.03 & $-0.23^{* * *}$ & 0.00 & 0.02 & 0.02 \\
\hline & $(0.02)$ & $(0.06)$ & $(0.01)$ & $(0.03)$ & $(0.02)$ \\
\hline \multirow[t]{2}{*}{ Gini } & -0.01 & 0.11 & -0.01 & $-0.10^{* * *}$ & $-0.10^{* * *}$ \\
\hline & $(0.02)$ & $(0.07)$ & $(0.00)$ & $(0.02)$ & $(0.02)$ \\
\hline \multicolumn{6}{|l|}{ Adjusted models } \\
\hline \multirow[t]{2}{*}{ Rank-rank } & -0.01 & $0.21^{* *}$ & 0.01 & $0.09^{* * *}$ & $0.08^{* * *}$ \\
\hline & $(0.02)$ & $(0.08)$ & $(0.01)$ & $(0.03)$ & $(0.02)$ \\
\hline \multirow[t]{2}{*}{ Upward mobility $\times-1$} & -0.01 & -0.07 & -0.01 & -0.02 & -0.01 \\
\hline & $(0.03)$ & $(0.10)$ & $(0.01)$ & $(0.03)$ & $(0.03)$ \\
\hline \multirow[t]{2}{*}{ Gini } & 0.03 & -0.07 & -0.00 & $-0.07^{*}$ & $-0.07^{* * *}$ \\
\hline & $(0.03)$ & $(0.10)$ & $(0.01)$ & $(0.03)$ & $(0.02)$ \\
\hline Individuals & 8810 & 8810 & 8810 & 8810 & 8810 \\
\hline
\end{tabular}

Each coefficient represents a model. Coefficients and standard errors are combined estimates from 20 multiple imputed datasets. Analyses based on exposure from 12 to 20 years old. We estimate different models depending on the outcome: Ordinal regression (self-reported health), General linear model (BMI, depression), Logistic regression (smoking), Quasi-Poisson regression (days smoking last month). ${ }^{* * *} p<0.001,{ }^{* *} p<0.01,{ }^{*} p<0.05$ 
Table S11: NLSY97 Stabilized treatment weights

\begin{tabular}{lcccccc}
\hline & & & \multicolumn{5}{c}{ Percentiles } \\
\cline { 5 - 7 } Weight & Mean & SD & 1 st & 25th & 75 th & 99 th \\
\hline Continuous exposure & & & & & & \\
Rank-rank & 1.01 & 0.91 & 0.35 & 0.71 & 1.05 & 3.57 \\
Upward mobility & 0.99 & 0.63 & 0.34 & 0.74 & 1.05 & 3.30 \\
Gini & 1.05 & 1.78 & 0.29 & 0.70 & 1.08 & 4.29 \\
Categorical (quintile) exposure & & & & & \\
Rank-rank & 1.00 & 0.56 & 0.39 & 0.68 & 1.09 & 3.51 \\
Upward mobility & 1.00 & 0.55 & 0.47 & 0.69 & 1.08 & 3.10 \\
Gini & 1.00 & 0.55 & 0.47 & 0.67 & 1.07 & 3.02 \\
\hline
\end{tabular}

Analyses based on exposure from 12 to 20 years old. Statistics based on 20 multiple imputed datasets. 


\subsubsection{PSID}

Table S12: Estimates of average continuous exposure on health indicators, PSID

\begin{tabular}{|c|c|c|c|c|c|}
\hline & Health status & BMI & Depression & Smoking & Cigarettes smoked \\
\hline \multicolumn{6}{|l|}{ Unadjusted models } \\
\hline \multirow[t]{2}{*}{ Rank-rank } & $-0.14^{*}$ & 0.58 & 0.02 & 0.13 & 0.10 \\
\hline & $(0.07)$ & $(0.30)$ & $(0.03)$ & $(0.11)$ & $(0.11)$ \\
\hline \multirow[t]{2}{*}{ Upward mobility $\times-1$} & $-0.14^{*}$ & 0.47 & 0.02 & -0.05 & -0.03 \\
\hline & $(0.07)$ & $(0.28)$ & $(0.03)$ & $(0.14)$ & $(0.12)$ \\
\hline \multirow[t]{2}{*}{ Gini } & -0.01 & 0.18 & -0.01 & -0.12 & $-0.13^{*}$ \\
\hline & $(0.08)$ & $(0.22)$ & $(0.02)$ & $(0.08)$ & $(0.07)$ \\
\hline \multicolumn{6}{|l|}{ Adjusted models } \\
\hline \multirow[t]{2}{*}{ Rank-rank } & -0.06 & 0.34 & 0.03 & 0.10 & 0.04 \\
\hline & $(0.08)$ & $(0.28)$ & $(0.03)$ & $(0.12)$ & $(0.11)$ \\
\hline \multirow[t]{2}{*}{ Upward mobility $\times-1$} & -0.07 & 0.58 & 0.07 & -0.05 & -0.09 \\
\hline & $(0.10)$ & $(0.32)$ & $(0.04)$ & $(0.14)$ & $(0.14)$ \\
\hline \multirow[t]{2}{*}{ Gini } & -0.02 & 0.27 & -0.02 & -0.15 & $-0.22^{*}$ \\
\hline & $(0.12)$ & $(0.29)$ & $(0.03)$ & $(0.13)$ & $(0.09)$ \\
\hline Individuals & 2273 & 2273 & 2273 & 2273 & 2273 \\
\hline
\end{tabular}

Each coefficient represents a model. Coefficients and standard errors are combined estimates from 20 multiple imputed datasets. Analyses based on exposure from 1 to 20 years old. We estimate different models depending on the outcome: Ordinal regression (self-reported health), General linear model (BMI, depression), Logistic regression (smoking), QuasiPoisson regression (cigarettes smoked). ${ }^{* *} p<0.001,{ }^{* *} p<0.01,{ }^{*} p<0.05$ 
Table S13: Estimates of average categorical (quintile) exposure on health indicators, PSID

\begin{tabular}{|c|c|c|c|c|c|}
\hline & Health status & BMI & Depression & Smoking & Cigarettes smoked \\
\hline \multicolumn{6}{|l|}{ Unadjusted models } \\
\hline \multirow[t]{2}{*}{ Rank-rank } & -0.07 & $0.37^{*}$ & 0.01 & 0.06 & 0.06 \\
\hline & $(0.05)$ & $(0.18)$ & $(0.02)$ & $(0.07)$ & $(0.07)$ \\
\hline \multirow[t]{2}{*}{ Upward mobility $\times-1$} & 0.09 & -0.33 & -0.01 & -0.01 & -0.03 \\
\hline & $(0.05)$ & $(0.20)$ & $(0.02)$ & $(0.08)$ & $(0.07)$ \\
\hline \multirow[t]{2}{*}{ Gini } & 0.01 & 0.08 & -0.00 & -0.08 & -0.09 \\
\hline & $(0.05)$ & $(0.15)$ & $(0.01)$ & $(0.06)$ & $(0.05)$ \\
\hline \multicolumn{6}{|l|}{ Adjusted models } \\
\hline \multirow[t]{2}{*}{ Rank-rank } & -0.04 & 0.16 & 0.03 & 0.07 & 0.05 \\
\hline & $(0.05)$ & $(0.17)$ & $(0.02)$ & $(0.07)$ & $(0.07)$ \\
\hline \multirow[t]{2}{*}{ Upward mobility $\times-1$} & 0.07 & -0.33 & -0.02 & -0.15 & -0.09 \\
\hline & $(0.06)$ & $(0.22)$ & $(0.02)$ & $(0.08)$ & $(0.07)$ \\
\hline \multirow[t]{2}{*}{ Gini } & 0.07 & 0.06 & -0.00 & -0.03 & -0.07 \\
\hline & $(0.06)$ & $(0.18)$ & $(0.02)$ & $(0.07)$ & $(0.06)$ \\
\hline Individuals & 2273 & 2273 & 2273 & 2273 & 2273 \\
\hline
\end{tabular}

Each coefficient represents a model. Coefficients and standard errors are combined estimates from 20 multiple imputed datasets. Analyses based on exposure from 1 to 20 years old. We estimate different models depending on the outcome: Ordinal regression (self-reported health), General linear model (BMI, depression), Logistic regression (smoking), QuasiPoisson regression (cigarettes smoked). ${ }^{* * *} p<0.001,{ }^{* *} p<0.01,{ }^{*} p<0.05$ 
Table S14: PSID Stabilized treatment weights

\begin{tabular}{lcccccc}
\hline & & & \multicolumn{5}{c}{ Percentiles } \\
\cline { 5 - 7 } Weight & Mean & SD & 1 st & 25th & 75 th & 99 th \\
\hline Continuous exposure & & & & & & \\
Rank-rank & 1.02 & 0.81 & 0.36 & 0.69 & 1.07 & 4.39 \\
Upward mobility & 1.04 & 2.66 & 0.30 & 0.57 & 1.04 & 4.50 \\
Gini & 1.08 & 1.96 & 0.24 & 0.66 & 1.06 & 5.52 \\
Categorical (quintile) & exposure & & & & & \\
Rank-rank & 1.00 & 0.61 & 0.41 & 0.69 & 1.07 & 3.40 \\
Upward mobility & 0.98 & 0.70 & 0.46 & 0.59 & 0.98 & 3.30 \\
Gini & 1.01 & 0.65 & 0.44 & 0.64 & 1.05 & 3.73 \\
\hline
\end{tabular}

Analyses based on exposure from 1 to 20 years old. Statisticis based on 20 multiple imputed datasets. 


\section{References}

van Buuren, S. (2018). Flexible Imputation of Missing Data (Second Edition). Chapman and Hall/CRC. 